\title{
Bradycardia-Induced Recurrent Torsade de Pointes: When Serenity Turns into Chaosity
}

\section{Check for updates}

DOI: $10.21859 / \mathrm{ijcp}-03045$

\section{Laksmi S. Agusta ${ }^{1,}$, Harnanik P. Riswati ${ }^{1}$, Rizal R. Akbar ${ }^{1}$, Ardian Rizal $^{2}$}

${ }^{1}$ General Practitioner of Wava Husada Hospital, Malang, East Java, Indonesia

${ }^{2}$ Cardiologist and Arrythmia Consultant, Wava Husada Hospital, Malang, East Java, Indonesia

${ }^{*}$ Corresponding author: Laksmi S. Agusta, General Practitioner of Wava Husada Hospital, Malang, East Java, Indonesia. E-mail: laksmisenja@gmail.com

\begin{tabular}{|c|c|}
\hline Submitted: $30-10-2018$ & \multirow{9}{*}{$\begin{array}{l}\text { Abstract } \\
\text { Torsade De Pointes is typical form of polymorphic ventricular tachycardia. It was in } \\
\text { the setting of bradycardia when first described. We present a case of patient coming to } \\
\text { emergency room with torsade de pointes development who was found to have } \\
\text { bradycardia on basal electrocardiography record. In fact, bradycardia has been shown } \\
\text { as a cause of acquired long QT syndrome that can lead to torsade de pointes. The } \\
\text { inverse relationship between heart rate and repolarization time primarily accounts for } \\
\text { QT prolongation. Finally, proper treatment considering electrophysiology mechanism } \\
\text { is essential to prevent mortality. }\end{array}$} \\
\hline Accepted: $03-12-2018$ & \\
\hline Keywords: & \\
\hline Torsade de Pointes & \\
\hline Long QT Syndrome & \\
\hline Tachycardia & \\
\hline Bradycardia & \\
\hline Tachycardia, Ventricular & \\
\hline $\begin{array}{l}\text { (c) 2018. International Journal of } \\
\text { Cardiovascular Practice. }\end{array}$ & \\
\hline
\end{tabular}

\section{INTRODUCTION}

Torsade de pointes (TDP) is a type of polymorphic ventricular tachycardia (VT), distingushed by undulations and twist of QRS complex over several beat around the baseline [1] A QT-prolongation is invariably present. It is indeed a potentially life threatening arrhythmia since it could degenerate into ventricular fibrillation. Bradycardia has been linked with development of VT reported in several experimental and clinical studies, including TDP. In fact, TDP was in the setting of bradycardia when first described by Dessertenne in 1966. TDP has been associated in many conditions related with bradycardia, such as atrioventricular block, drugs, vagotonia or hypothyroidism.

\section{CASE PRESENTATION}

A 78-year-old woman presented in emergency room (ER) with several episodes of faintness for two days prior. She was feeling weak, unwell, accompanied by diaphoresis and shortness of breath. Her past medical history was significant for diabetes mellitus which was irregularly controlled.

Physical examination finding were Glasgow Coma Scale) score of 15 , blood pressure of $177 / 80$, respiratory rate of 22 , temperature of $36.8^{\circ} \mathrm{C}$, oxygen saturation of $98 \%$, and radial pulse of 58 beats per minutes regular. Head / neck, chest, abdomen, and extremity examination were unremarkable.

Not a long time after arrival, the patient suddenly became apnea. Assisted ventilation was performed, and spontaneous breathing restored. Electrocardiogram (ECG) showed multiple ventricular ectopic beats followed by polymorphic VT which was later selfterminated (Fig 1). Magnesium Sulphate (MgSO4) intravenous infusion with a dose of 2 gram over 30 minutes continued by 0.5 gram per hour was administrated for the suppression of VT.

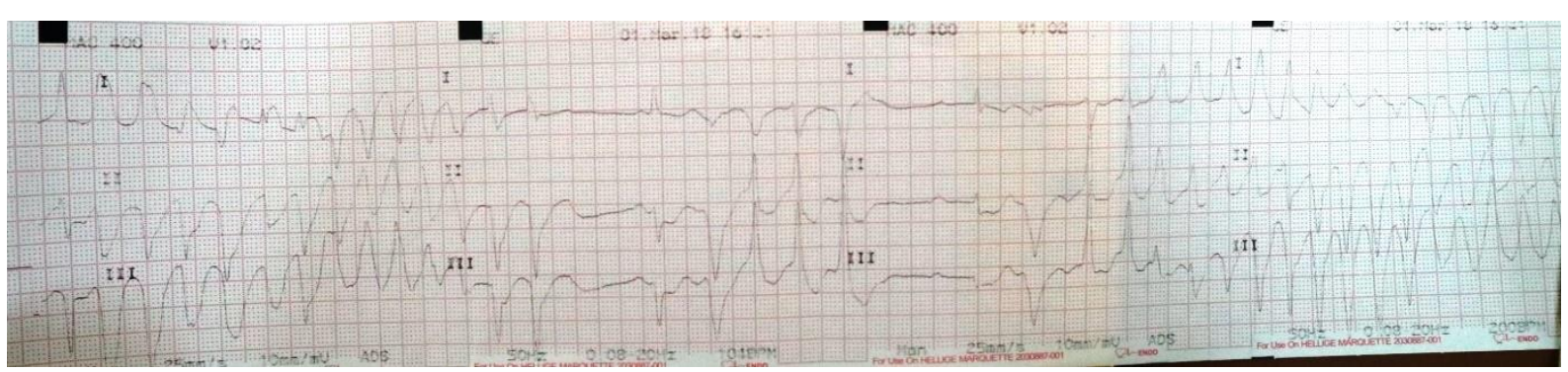

Figure 1: Initial Electrocardiography Record 
It was after 30 minutes later, the heart rate was dropping to 40 beats per minutes. Profound bradycardia was observed (Fig 2). Sulfas atropine $0.5 \mathrm{mg}$ was directly given and followed by dopamine infusion of 5 $\mathrm{mcg} / \mathrm{kg} /$ minutes.

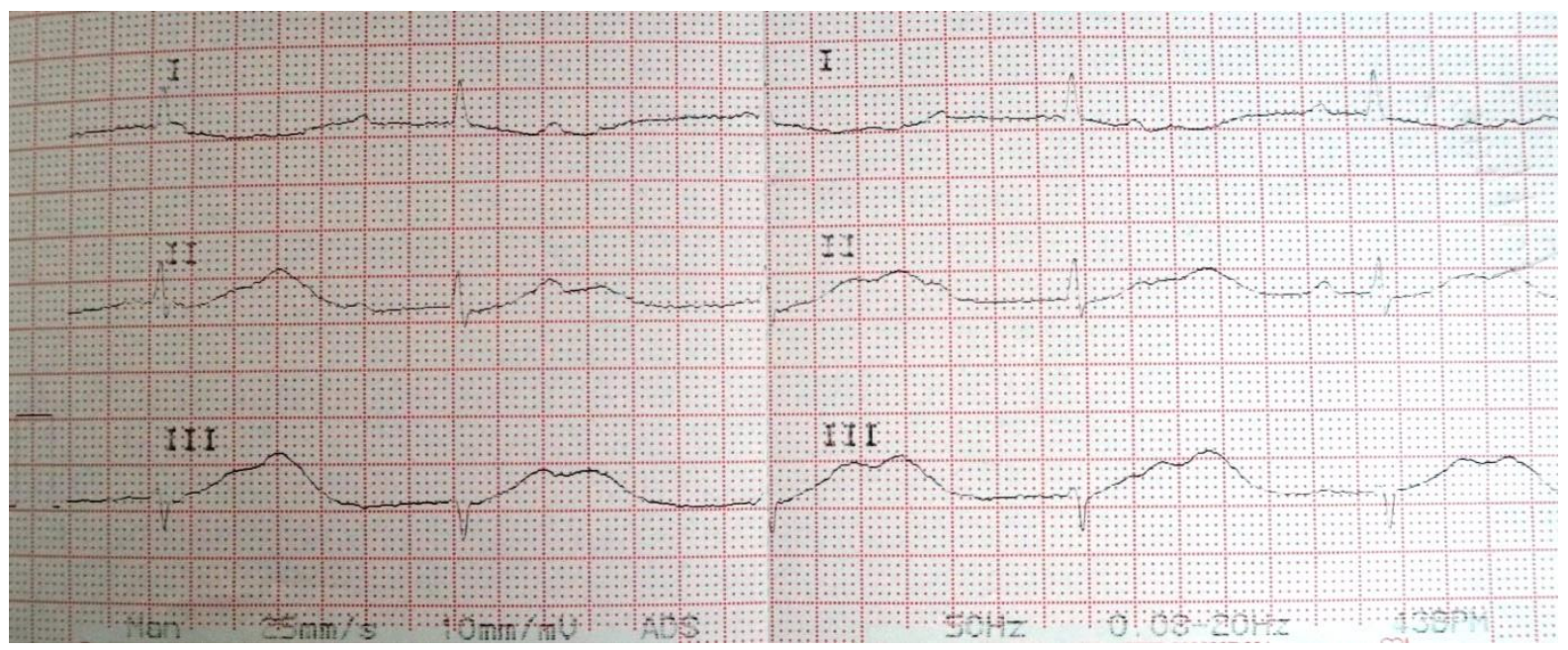

Figure 2: Profound Bradycardia was observed during Emergency Room Observation

Several episodes of polymorphic VT were developed during ER observation. Electrical cardioversion of 100 200 joule was performed and promptly converted the rhythm back to sinus. But the polymorphic VT continued to develop repeatedly for more than ten episodes even after in intensive care unit (ICU) observation (Fig 3).

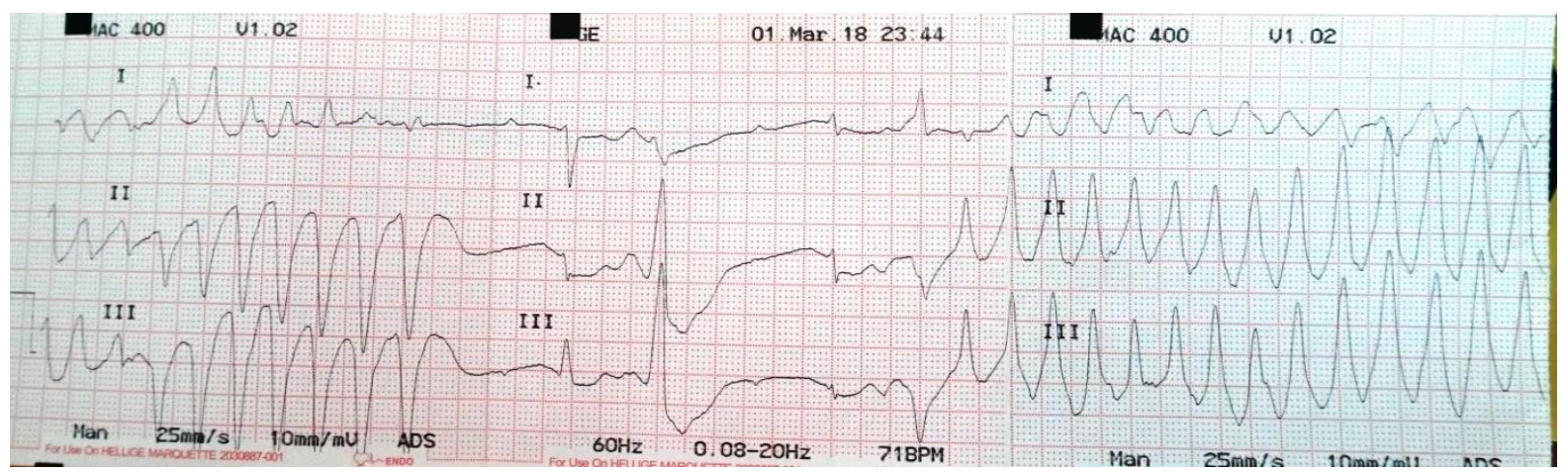

Figure 3: Repeated Episodes of Torsade de Pointes was developed during Intensive Care Unit Observation

Laboratory finding demonstrated an elevated blood glucose level $(432 \mathrm{mg} / \mathrm{dL})$ and low kalium serum level $(2.70 \mathrm{mmol} / \mathrm{L})$ which were managed by intravenous short acting insulin of $3 \times 4$ iu and Kalium Chloride infusion of 50 meq over 24 hours. Basal ECG revealed bradycardia of 40 beats per minutes with sinus arrest and junctional escape rythm (Fig 4). Temporary pacemaker implantation was considered to be the next treatment.

\section{DISCUSSION}

TDP involves electrophysiological mechanism by inhibition of the delayed rectifier potassium current. This condition results in excessive number of positive ions within the cellular membrane causing a prolonged repolarization phase, reflecting as QT interval in ECG record [2].

This slow heart rate apparently shown to be responsible in causing acquired long QT syndrome which consequently leads to TDP. Repolarization time in myocardial tissue depends on the previous cycle length. A long duration of cycle in bradycardia will be followed by an extent in repolarization time, which is regarding as vulnerable period. The inverse relationship between heart rate and repolarization time primarily accounts for QT prolongation [3]. In this case, the value of corrected-QT (QTc) interval was $660 \mathrm{~ms}$. In fact, this prolonged repolarization is frequently heterogenous and leads to a dispersion of repolarization. This vulnerable period eventually gives rise for early after depolarization (EAD) and reentry [4]. EAD may manifest on the ECG as tall $U$ waves and become premature ventricular contraction (PVC) if reaches amplitude threshold. TDP is trigerred when an ectopic beat occurs during the prolonged repolarization phase. It is known as ' $\mathrm{R}$ on $\mathrm{T}$ ' phenomenon, which is notable on patient's ECG record preceded the episodes of TDP. 
Different risk factors have been associated to TDP besides bradycardia. Amongst, the most commonly described are female gender and elderly age, which are illustrated on this 78-year-old-woman case. In general population, women tend to have a longer QTc interval than men. Furthermore, elderly persons are more likely vulnerable of co-morbid conditions like hypertension, coronary artery disease and heart failure, and these are known to result in down regulation of potassium channels.

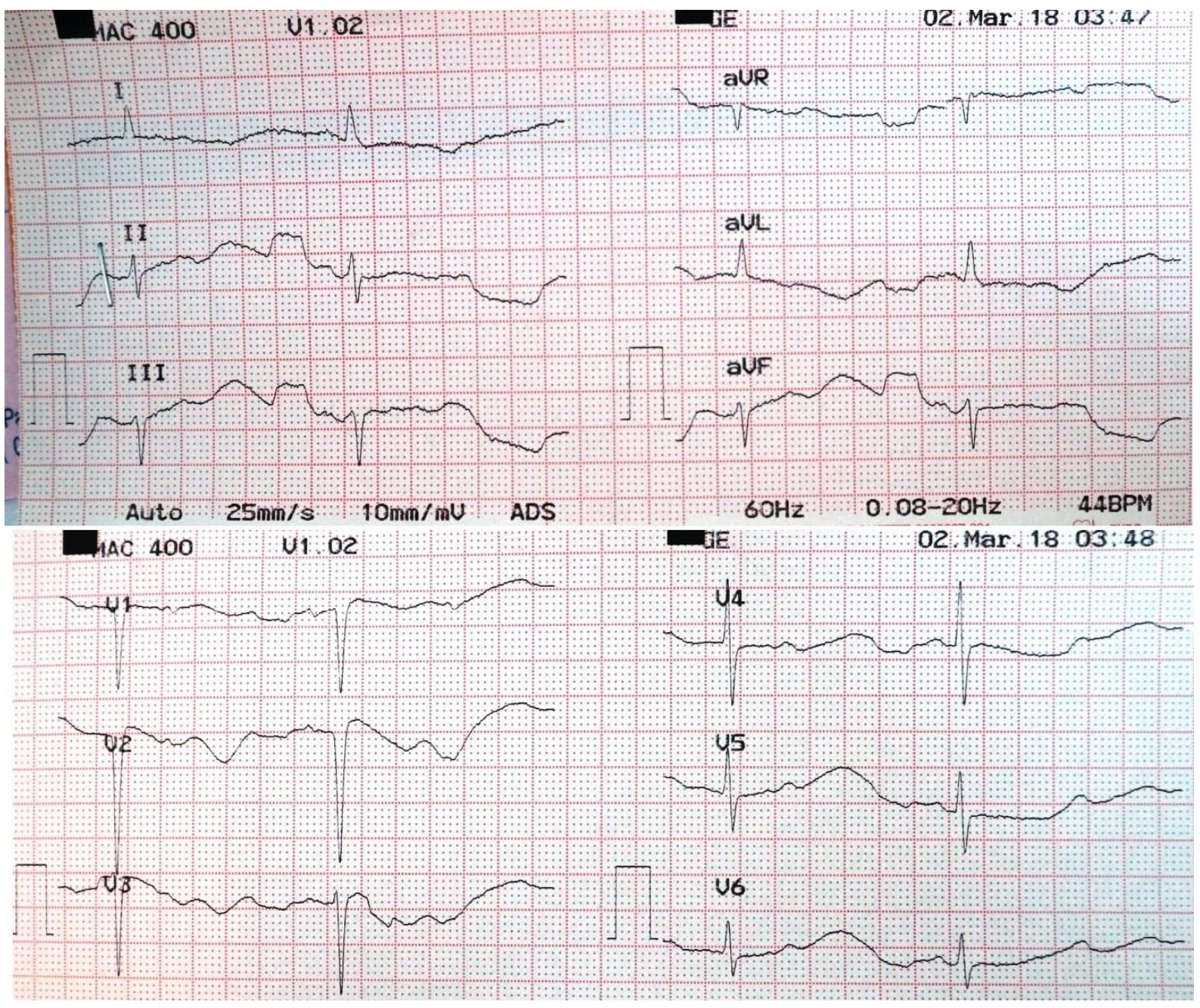

Figure 4: Basal Electrocardiography

Laboratory finding represents low kalium serum level and elevated blood glucose level. Some studies have also described electrolytic imbalances, including hypokalemia, as TDP facilitators. Hyperglycemia has also been frequently associated with QTc prolongation in patient suffering acute illnesses [5].

This two conditions should be managed immediately but carefully since treatment of hyperglycemia can result as hypokalemia as well and excessive correction of hypokalemia can lead to another malignant arrythmia. We assesed this patient having symptomatic bradycardia due to sinus nodal dysfunction and subsequent bradicardia-induced TDP. We cannot exclude the possibility of long QT syndrome as differential diagnosis since the patient had obvious QTc prolongation, even though it was less likely as she was elderly and did not have any supporting history.
We used intravenous $\mathrm{MgSO}_{4}$ as arrythmia supression since it is recommended as the immediate first line therapy for TDP. Magnesium inhibits the late calcium influx via L-type calcium channels associating with delayed ventricular repolarization, hence reducing $\mathrm{EAD}$ amplitude [6]. As a result, EAD is unlikely to reach threshold potential and trigger TDP. We also administered sulfas atropin and dopamin infusion to maintain heart rate and haemodinamic stability.

As definitive treatment, besides the fact that this patient had an inadequate respons to pharmacological treatment, this patient was offered to undergo pacemaker implantation. Indonesian Heart Association guideline of Cardiovascular Implantable Electronic Devices favors pacemaker implantation as indicated for symptomatic bradycardia due to sinus node dysfunction [7]. 
Pacing is generally took into account as an option after magnesium sulphate. The increase of heart rate due to transvenous pacing will prevent pauses and suppress episodes of TDP. Moreover, a study involving patients who ranged in age from 18 months to 80 years on initial presentation with TDP has shown a normalization in QT interval and complete suppression of TDP after implantation of ventricular pacing at appropriate rates. 1

\section{CONCLUSIONS}

This case is an excellent example of recurrent TDP in the setting of bradycardia accompanied by highly associated risk factors. Proper and immediate treatment considering electrophisiology mechanism should be attempted as a consequence to prevent mortality.

\section{REFERENCES}

1. Philippe C, Alina S. Atrio-Ventricular Block-Induced Torsades de Pointes: An Update. Aspects of Pacemakers-Functions and Interactions in Cardiac and Non-Cardiac Indications: InTech; 2011.
2. Cohagan B, Brandis D. Torsade de Pointes: Stat Pearls Publishing; 2017.

3. Namboodiri N. Bradycardia-induced Torsade de Pointes - An arrhythmia Less Understood. Indian Pacing Electrophysiol J. 2010;10(10):435-8.

www.ncbi.nlm.nih.gov/pubmed/21151381

4. Haverkamp W, Breithard G, Camm A. The potential for QT prolongation and proarrhythmia by non-antiarrhythmic drugs: clinical and regulatory implications. Reports on a policy conference of the European Society of Cardiology. Cardiovasc Res. 2000;47:219-33. http://dx.doi.org/10.1016/S00086363(00)00119-X

5. Pickham D, Flowers E, Drew BJ. Hyperglycemia is associated with corrected QT prolongation and mortality in acutely ill patients. J Cardiovasc Nurs. 2014;29(3):264-70. http://dx.doi.org/10.1097/JCN.0b013e31827f174c www.ncbi.nlm.nih.gov/pubmed/23364575

6. Thomas SH, Behr ER. Pharmacological treatment of acquired QT prolongation and torsades de pointes. Br J Clin Pharmacol. 2016;81(3):420-7. http://dx.doi.org/10.1111/bcp.12726 www.ncbi.nlm.nih.gov/pubmed/26183037

7. Hanafy D, Yuniadi Y, Raharjo S, Tondas A, Rahadian A, Yamin M, et al. Pedoman Terapi Memakai Alat Elektronik Kardiovaskular Implan (Aleka) Perhimpunan Dokter Spesialis Kardiovaskular Indonesia 2014. Indonesian J Cardiol. 2014:171-245. 